\title{
TiN Nanoporous Electrode covered by Single Cell as Bio-Electronic Sensor of Radiation Hazard
}

\author{
(C) D.D. Stupin \\ St. Petersburg Academic University, Russian Academy of Sciences, \\ 194021 St. Petersburg, Russia \\ E-mail: Stu87@ya.ru, Stupin@spbau.ru
}

Here we demonstrate a simple label-free bio-electronic radiation (ultra-violet) hazard detector based on non-destructive electrical impedance spectroscopy technique with a single living cell as a sensing element. Radiation results in wrong cell functioning and, as corollary, cells' membrane distortion. The latter results in impedance shift for the electrode covered by the cell. The additional sensitivity of impedance shift is achieved by the simultaneous usage of the TiN porous electrodes and adaptive filtering for impedance data processing. The main advantage of the proposed sensor with respect to purely physical and/or chemical sensors is high selectivity: it a priory reacts only on the dangerous for living beings radiation.

\section{Acknowledgments}

The author acknowledges Nikolay A. Verlov, Nikolay A. Knyazev, Sergei V. Koniakhin and Michael V. Dubina for multifaceted assistance and support.

This study was funded by Ministry of Education and Science of Russian Federation, governmental order N 16.9790.2017/BCh. 